\section{New Books from North-Holland}

\section{Currents in Hadron Physics}

By V. ALFARO, University of Torino, V.S. FUBINI, Massachusetts Institute of Technology and University of Torino, G. FURLAN, University of Trieste, and C. ROSSETTI, University of Torino.

\section{3. approx. 890 pages}

Dfl. 250.00 (about $\$ \mathbf{8 7 . 7 0}$ )

This book aims to assist the newcomer to the field of theoretical elementary particle physics in becoming acquainted with the present state of knowledge and the trends of progress. The most important facts and techniques developed in the last years in hadron physics have been collected together with particular emphasis given to the achievements going under the name of current algebra.

\section{CONTENTS}

Introduction to the theory of strong interactions. Weak and electromagnetic currents. Ward identities and low-energy limits. Dispersion sum rules in current algebra. Phenomenological Lagrangians and chiral symmetry. Completeness sum rules from equaltime commutators. The role of Lorentz invariance. The infinite-momentum limit. A new approach to strong interactions: duality. Open problems and recent developments in the theory of current. Current commutators on the light cone. References.

\section{Fundamental Interactions} and the Nucleus

By R.J. BLIN-STOYLE, University of Sussex England

\section{362 pages}

Dfl. 60.00 (about \$21.10)

This book provides a fairly detailed, up-to date account of the different aspects of the fundamental interactions of elementary particles.

CONTENTS: Fundamental interactions and the nucleus. Nuclear $\beta$ - decay. The polar vecto current in $\beta$ - decay. The axial vector curren in $\beta$ - decay. Unusual currents and interactions in $\beta$ - decay. The lepton current and the neutrino $\mu$ - capture. The weak internucleon potential. The effect of a parity violating potential in low energy nuclear physics processes. Time reversal non-invariance in nuclear reactions and electromagnetic transitions. Electromagnetic and charge dependen interactions. Strong interactions in the nucleus. Appendices.

\section{NORTH ${ }^{N}$ HOLLAND}

P.O. Box 211 - Amsterdam - The Netherlande

Distributed in the United States and Canada by American Elsevier Publishing Company Inc. 52 Vanderbilt Avenue, New York, N.Y. 10017

\title{
III International Symposium on Toroidal Plasma Confinement
}

\author{
26-30 March 1973, Garching, Fed. Rep. of Germany
}

The third International Symposium on Toroidal Plasma Confinement, held at Garching, 26-30 March 1973, was sponsored by the European Physical Society and organized by the MaxPlanck-Institut für Plasmaphysik. This Symposium which has followed those of Princeton (1966) and Dubna (1969), displayed the strong increase in emphasis on toroidal confinement within the field of thermonuclear research during recent years. Not only were there close to 200 participants from all over the world, but the communications (about 70) and discussions covered a large part of the activity in fusion-oriented plasma physics.

The programme included sessions on toroidal pinches, Tokamaks, internal ring devices, Stellarators, heating of and neutral injection into toroidal devices, theory of toroidal confinement, and planned experiments. There was also an opportunity of visiting the facilities of the Max-Planck-Institut für Plasmaphysik.

In the Tokamak line, on which a large part of the effort in toroidal confinement is concentrated, new results reported indicate that, by going to higher plasma currents and current densities, performance can be further improved. Impurities seem to play an important part in limiting the energy confinement, which calls for measures to decrease the interaction of the plasma with the wall material. Adiabatic compression of a Tokamak discharge has been shown to yield effective plasma heating by the Princeton and Leningrad groups. The possibilities offered by using a non-circular cross-section were discussed, and preliminary results of an experiment of this type were reported by the group of the Kurchatov Institute (Moscow). Results obtained from the Princeton internal ring device indicate that the confinement, which for lower temperatures follows typically a 'classical' scaling, is impaired at higher temperatures, probably because of plasma turbulence. In the field of Stellarators, a new way of building such devices using shaped coils instead of helical windings was reported by the Garching group.

The meeting was well organized and was run as informally as possible and with as much precision as necessary, and these factors contributed much to its success.

\section{F. Engelmann}

\section{Advanced Seminar on Condensed Matter in Astrophysics}

\section{December 1972 - 5 January 1973, Arad, Israel}

This seminar, one of a series of Israel Scientific Research Conferences, was sponsored by the Israel National Council for Research and Development. It was held between 27 December 1972 and 5 January 1973 in Arad, Israel with around 60 participants - astrophysicists and solid state physicists.

A general introduction to stellar evolution was presented by E. E. Salpeter (Cornell University). He followed this up by a number of talks on basic theories of equations of state, with special emphasis on the Jovian planets. G. Baym (University of Illinois) gave a detailed discussion of neutron star structure, superfluidity and super- conductivity. Compact X-ray sources and starquakes were described by D. Pines (University of Illinois) and J. Ostriker (Princeton University Observatory). E. Schatzman (Observatoire de Paris) gave a series of talks on white dwarfs. A. Cameron (Yeshiva University) discussed the problem of nucleosynthesis in connection with supernovae mechanisms, and the intriguing problem of solar neutrinos. Pulsar observations and theories were reviewed by J. Ostriker and by M. Ruderman (Columbia University), who also dealt with the properties of matter in strong magnetic fields.

\section{E. Schatzman, G. Rakavy}

